\title{
Infinite propagation speed for the two component $b$-family system
}

Zhiqiang Wei ${ }^{i^{*}}$, Yanyi Jin² and Liangbing $\mathrm{Jin}^{2}$

\section{"Correspondence:}

wei.zhiqiang@yahoo.com

${ }^{1}$ School of Mathematics and Information Sciences, North China

University of Water Resources and Electric Power, Zhengzhou, 450011,

China

Full list of author information is available at the end of the article

\begin{abstract}
In this paper, we propose the infinite propagation speed for the two component $b$-family system. No matter what the profile of the compactly supported initial datum $\left(u_{0}(x), \rho_{0}(x)\right)$ is, for any $t>0$ in its lifespan, the solution $u(x, t)$ is positive at infinity and negative at negative infinity.

MSC: 37L05; 35Q58; 26A12
\end{abstract}

Keywords: two component $b$-family system; infinite propagation speed

\section{Introduction}

In this essay, we consider the following model, named two-component $b$-family system

$$
\begin{cases}m_{t}=u m_{x}+k_{1} m u_{x}+k_{2} \rho \rho_{x}, & t>0, x \in \mathbb{R}, \\ \rho_{t}=k_{3}(\rho u)_{x}, & t>0, x \in \mathbb{R},\end{cases}
$$

where $m=u-u_{x x}$. As far as we known, it seems that system (1.1) appears initially by Guha in [1]. There are two cases about this system (i) $k_{1}=b, k_{2}=2 b$ and $k_{3}=1$; (ii) $k_{1}=b+1$, $k_{2}=2$ and $k_{3}=b$ with $b \in \mathbb{R}$. In [2], they applied Kato's theory [3] to establish the local well-posedness for the Cauchy problem of (1.1). It is proved that there exists a unique solution $(u, \rho) \in C\left([0, T) ; H^{s} \times H^{s-1}\right)$ for any $\left(u_{0}, \rho_{0}\right) \in H^{s} \times H^{s-1}$ with $s>\frac{3}{2}$. The precise blow-up scenarios and some blow-up criteria were also established in [2].

We only consider the case $k_{3}=1$,

$$
\begin{cases}m_{t}=u m_{x}+k_{1} m u_{x}+k_{2} \rho \rho_{x}, & t>0, x \in \mathbb{R}, \\ \rho_{t}=(\rho u)_{x}, & t>0, x \in \mathbb{R} .\end{cases}
$$

Obviously, under the constraint of $\rho=0$, system (1.2) reduces to the $b$-family equations

$$
m_{t}+u m_{x}+b m u_{x}=0
$$

which were derived physically by Holm and Staley in [4]. Detailed description of the corresponding strong solutions to (1.3) with $u_{0}$, being its initial data, was given by Zhou in [5]. He established a sufficient condition in profile on the initial data for blow-up in finite time. The necessary and sufficient condition for blow-up is still a challenging problem for us at present. More precisely, Theorem 3.1 in [5] means that no matter what the profile of

\section{㩏 Springer}

(c) 2013 Wei et al.; licensee Springer. This is an Open Access article distributed under the terms of the Creative Commons Attribution License (http://creativecommons.org/licenses/by/2.0), which permits unrestricted use, distribution, and reproduction in any medium, provided the original work is properly cited. 
the compactly supported initial datum $u_{0}(x)$ is (no matter whether it is positive or negative), for any $t>0$ in its lifespan, the solution $u(x, t)$ is positive at infinity and negative at negative infinity, it is really a very nice property for the $b$-family equations. For $b=2$ and $b=3,(1.3)$ is the famous Camassa-Holm equation [6] and Degasperis-Procesi equation [7], respectively. Many papers [8-14] are devoted to their studies.

Another related system is the two component Camassa-Holm system. Recently, Constantin and Ivanov in [15] gave a demonstration about its derivation in view of the fluid shallow water theory from the hydrodynamic point of view. This generalization, similarly to the Camassa-Holm equation, possessed the peakon, multi-kink solutions and the biHamiltonian structure [16] and is integrable. Well-posedness and wave breaking mechanism were discussed in [17], and the existence of global solutions was analyzed in [18]. The infinite propagation speed was studied by Henry in [19].

In the following section, we will show our main results and give the detailed proof.

\section{Main results}

Motivated by Mckean's deep observation for the Camassa-Holm equation [11], we can do the similar particle trajectory as

$$
\begin{cases}q_{t}=-u(q, t), & 0<t<T, x \in \mathbb{R}, \\ q(x, 0)=x, & x \in \mathbb{R},\end{cases}
$$

where $T$ is the life span of the solution, then $q$ is a diffeomorphism of the line. Differentiating the first equation in (2.1) with respect to $x$, one has

$$
\frac{d q_{t}}{d x}=q_{x t}=-u_{x}(q, t) q_{x}, \quad t \in(0, T)
$$

Hence

$$
q_{x}(x, t)=\exp \left\{\int_{0}^{t}-u_{x}(q, s) d s\right\}, \quad q_{x}(x, 0)=1
$$

Our first result will show that $m$ and $\rho$ have compact support if their initial data have this property. $m$ and $\rho$ are the solutions of system (1.2).

Theorem 2.1 Assume that $m_{0}=u_{0}-u_{0 x x}$ has compact support, contained in the interval $\left[\alpha_{m_{0}}, \beta_{m_{0}}\right]$, and that $\rho_{0}$ is also compactly supported, with support contained in $\left[\alpha_{\rho_{0}}, \beta_{\rho_{0}}\right]$. If $T=T\left(u_{0}, \rho_{0}\right)>0$ is the maximal existence time of the unique classical solutions $(u, \rho)$ to the system (1.2) with the given initial data $u_{0}(x)$ and $\rho_{0}(x)$, then for any $t \in[0, T), m(x, t)$ and $\rho(x, t)$ have compact support.

Proof By the particle trajectory defined in (2.1), we find that

$$
\begin{aligned}
\frac{d}{d t} m(q(x, t), t) q_{x}^{k_{1}}(x, t) & =m_{t} q_{x}^{k_{1}}+m_{x} q_{t} q_{x}^{k_{1}}+k_{1} m q_{x t} q_{x}^{k_{1}-1} \\
& =\left(\rho_{t}-u m_{x}-k_{1} m u_{x}\right) q_{x}^{k_{1}}=k_{2} \rho \rho_{x} q_{x}^{k_{1}}
\end{aligned}
$$


and

$$
\frac{d}{d t} \rho(q(x, t), t) q_{x}(x, t)=\rho_{t} q_{x}+\rho_{x} q_{t} q_{x}+\rho q_{x t}=\left(\rho_{t}-(u \rho)_{x}\right) q_{x}=0 .
$$

Therefore,

$$
\rho(q(x, t), t) q_{x}(x, t)=\rho_{0}\left(x_{0}\right)
$$

Since $q_{x} \geq 0$ and $\rho_{0}$ is compactly supported, it follows from this relation that $\rho(\cdot, t)$ is compactly supported for all times $t \in[0, T)$, with support contained in the interval $\left[q\left(\alpha_{\rho_{0}}, t\right), q\left(\beta_{\rho_{0}}, t\right)\right]$. Setting

$$
\alpha=\min \left\{\alpha_{m_{0}}, \alpha_{\rho_{0}}\right\}, \quad \beta=\max \left\{\beta_{m_{0}}, \beta_{\rho_{0}}\right\} .
$$

Due to

$$
\frac{d}{d t} m(q(x, t), t) q_{x}^{k_{1}}(x, t)=0, \quad \text { on } x \in \mathbb{R}-\left[\alpha_{\rho_{0}}, \beta_{\rho_{0}}\right]
$$

it follows that $m(\cdot, t)$ is compactly supported, with its support contained in the interval $[q(\alpha, t), q(\beta, t)]$, for all $t \in[0, T)$.

In order to prove Theorem 2.3, we will use the following result.

Lemma 2.2 [9] Let $u \in C^{2}(\mathbb{R}) \cap H^{2}(\mathbb{R})$ be such that $m=u-u_{x x}$ has compact support. Then $u$ has compact support if and only if

$$
\int_{\mathbb{R}} e^{x} m d x=\int_{\mathbb{R}} e^{-x} m d x=0
$$

Theorem 2.3 Let $k_{1} \in[0,3], k_{2} \geq 0$, assume that the function $u_{0}$ has compact support. Let $T \geq 0$ be the maximal existence time of the unique solution $u(x, t)$ with initial data $u_{0}(x)$. If at every $t \in[0, T), u(x, t)$ has compact support, then $u$ and $\rho$ are identically zero.

Proof Using (1) and differentiating the left hand side of (2.2) with respect to $t$ we get

$$
\begin{aligned}
\frac{d}{d t} \int_{\mathbb{R}} e^{x} m d x & =\int_{\mathbb{R}} e^{x} m_{t} d x=\int_{\mathbb{R}} e^{x} u m_{x} d x+\int_{\mathbb{R}} e^{x} k_{1} u_{x} m d x+\int_{\mathbb{R}} e^{x} k_{2} \rho \rho_{x} d x \\
& =\int_{\mathbb{R}} e^{x} u m_{x} d x+\int_{\mathbb{R}} e^{x} k_{1} u_{x} m d x+\int_{\mathbb{R}} e^{x} k_{2} \rho \rho_{x} d x \\
& =-\int_{\mathbb{R}} e^{x} u m d x+\int_{\mathbb{R}} e^{x}\left(k_{1}-1\right) u_{x} m d x-\int_{\mathbb{R}} e^{x} \frac{k_{2}}{2} \rho^{2} d x \\
& =\int_{\mathbb{R}} \frac{-k_{1}}{2} u^{2}+\frac{k_{1}-3}{2} u_{x}^{2}-\frac{k_{2}}{2} \rho^{2} d x,
\end{aligned}
$$

where all boundary terms after integration by parts vanish as both $m(\cdot, t)$ and, by assumption, $u(\cdot, t)$ have compact support for all $t \in[0, T)$.

The expression under the integral on the right hand side of this relation must be identically zero by (2.2). This implies that all of the terms in the bracket must be identically zero, and in particular $u=\rho=0$. This completes the proof. 
The theorem above means that if $u \neq 0$ is a function with compact support, then the classical solution $u(x, t)$ of system (1.2) must instantly lose the compactness of its support. The following theorem will give a detailed description about the profile of the solution $u(x, t)$.

Theorem 2.4 Let $0 \leq k_{1} \leq 3$ and $k_{2} \geq 0$, $u$ is a nontrivial solution of $(1.2)$. If $u_{0}(x)=u(x, 0)$ has compact support $\left[\alpha_{u_{0}}, \beta_{u_{0}}\right]$, and $\rho_{0}$ is also initially compactly supported, on the interval $\left[\alpha_{\rho_{0}}, \beta_{\rho_{0}}\right]$, then for $t \in(0, T]$, we have

$$
u(x, t)= \begin{cases}f_{-}(t) e^{-x}, & \text { for } x>q(\beta, t), \\ f_{+}(t) e^{x}, & \text { for } x<q(\alpha, t),\end{cases}
$$

where $f_{-}(t)$ and $f_{+}(t)$ denote continuous nonvanishing functions with $f_{-}(t)<0$ and $f_{+}(t)>0$ for $t \in(0, T]$. Furthermore, $f_{-}(t)$ is a strictly decreasing function, while $f_{+}(t)$ is increasing function.

Proof Since $u_{0}$ and $\rho_{0}$ are compactly supported. By Theorem 2.1, $m$ is compactly supported with its support contained in the interval $[q(\alpha, t), q(\beta, t)]$. Hence the following functions are well-defined:

$$
E(t)=\int_{\mathbb{R}} e^{x} m(x, t) d x \quad \text { and } \quad F(t)=\int_{\mathbb{R}} e^{-x} m(x, t) d x,
$$

with

$$
E_{0}=\int_{\mathbb{R}} e^{x} m_{0}(x) d x=0 \quad \text { and } \quad F_{0}=\int_{\mathbb{R}} e^{-x} m_{0}(x) d x=0 .
$$

Then for $x>q(\beta, t)$, we have

$$
u(x, t)=\frac{1}{2} e^{-|x|} * m(x, t)=\frac{1}{2} e^{-x} \int_{q(\alpha, t)}^{q(\beta, t)} e^{\tau} m(\tau, t) d \tau=\frac{1}{2} e^{-x} E(t)
$$

Similarly, when $x<q(\alpha, t)$, we get

$$
u(x, t)=\frac{1}{2} e^{-|x|} * m(x, t)=\frac{1}{2} e^{x} \int_{q(\alpha, t)}^{q(\beta, t)} e^{-\tau} m(\tau, t) d \tau=\frac{1}{2} e^{x} F(t) .
$$

Hence, as consequences of (2.3) and (2.4), we have

$$
u(x, t)=-u_{x}(x, t)=u_{x x}(x, t)=\frac{1}{2} e^{-x} E(t), \quad \text { as } x>q(\beta, t)
$$

and

$$
u(x, t)=u_{x}(x, t)=u_{x x}(x, t)=\frac{1}{2} e^{x} F(t), \quad \text { as } x<q(\alpha, t) .
$$

On the other hand,

$$
\frac{d E(t)}{d t}=\int_{\mathbb{R}} e^{x} m_{t}(x, t) d x
$$


It is easy to get

$$
m_{t}=u u_{x}-\left(u u_{x}\right)_{x x}+\partial_{x}\left(\frac{k_{1}}{2} u^{2}+\frac{3-k_{1}}{2} u_{x}^{2}\right)+k_{2} \rho \rho_{x} .
$$

Substituting the identity (2.7) into $d E(t) / d t$, we obtain

$$
\begin{aligned}
\frac{d E(t)}{d t} & =\int_{\mathbb{R}} e^{x}\left(u u_{x}-\left(u u_{x}\right)_{x x}+\partial_{x}\left(\frac{k_{1}}{2} u^{2}+\frac{3-k_{2}}{2} u_{x}^{2}\right)\right) d x+\int_{\mathbb{R}} e^{x}\left(k_{2} \rho \rho_{x}\right) d x \\
& =\int_{\mathbb{R}} e^{x}\left(\frac{-k_{1}}{2} u^{2}+\frac{k_{2}-3}{2} u_{x}^{2}-\frac{k_{2}}{2} \rho^{2}\right) d x,
\end{aligned}
$$

where we use (2.5) and (2.6).

Therefore, in the lifespan of the solution, we have

$$
E(t)=\int_{0}^{t} \int_{\mathbb{R}} e^{x}\left(\frac{-k_{1}}{2} u^{2}+\frac{k_{1}-3}{2} u_{x}^{2}-\frac{k_{2}}{2} \rho^{2}\right)(x, \tau) d x d \tau<0 .
$$

By the same argument, one can check that the following identity for $F(t)$ is true

$$
F(t)=\int_{0}^{t} \int_{\mathbb{R}} e^{-x}\left(\frac{k_{1}}{2} u^{2}+\frac{3-k_{1}}{2} u_{x}^{2}+\frac{k_{2}}{2} \rho^{2}\right)(x, \tau) d x d \tau>0
$$

In order to complete the proof, it is sufficient to let $f_{-}(t)=\frac{1}{2} E(t)$ and $f_{+}(t)=\frac{1}{2} F(t)$.

Remark 2.1 Theorem 2.3 means that no matter what the profile of the compactly supported initial datum $u_{0}(x)$ is (no matter whether it is positive or negative), for any $t>0$ in its lifespan, the solution $u(x, t)$ is negative at infinity and positive at negative infinity.

\section{Competing interests}

The authors declare that they have no competing interests.

\section{Authors' contributions}

ZW proposed the problems and finished the whole manuscript. YJ proved Theorems 2.1 and 2.3. LJ proved Theorem 2.4 All authors read and approved the final manuscript.

\section{Author details}

${ }^{1}$ School of Mathematics and Information Sciences, North China University of Water Resources and Electric Power, Zhengzhou, 450011, China. '2Department of Mathematics, Zhejiang Normal University, Jinhua, 321004, P.R. China.

\section{Acknowledgements}

This work is partially supported by ZJNSF (Grant No. LQ13A010008) and NSFC (Grant No. 11226176). Thanks are also given to the anonymous referees for careful reading and suggestions.

\section{Received: 20 June 2013 Accepted: 31 July 2013 Published: 14 August 2013}

\section{References}

1. Guha, P: Euler-Poincaré formalism of (two component) Degasperis-Procesi and Holm-Staley type systems. J. Nonlinear Math. Phys. 14, 398-429 (2007)

2. Liu, J, Yin, Z: On the Cauchy problem of a two-component $b$-family equation. Nonlinear Anal., Real World Appl. 12, 3608-3620 (2011)

3. Kato, T: Quasi-linear equations of evolution, with applications to partial differential equations. In: Spectral Theory and Differential Equations. Lecture Notes in Math, vol. 448, pp. 25-70. Springer, Berlin (1975)

4. Holm, D, Staley, M: Nonlinear balance and exchange of stability of dynamics of solitons, peakons, ramps/cliffs and leftons in a $1+1$ nonlinear evolutionary PDE. Phys. Lett. A 308, 437-444 (2003)

5. Zhou, Y: On solutions to the Holm-Staley b-family of equations. Nonlinearity 23, 369-381 (2010) 
6. Camassa, R, Holm, D: An integrable shallow water equation with peaked solitons. Phys. Rev. Lett. 71, 1661-1664 (1993)

7. Degasperis, A, Procesi, M: Asymptotic integrability. In: Degasperis, A, Gaeta, G (eds.) Symmetry and Perturbation Theory, pp. 23-37. World Scientific, Singapore (1999)

8. Constantin, A, Escher, J: Well-posedness, global existence and blow-up phenomena for a periodic quasilinear hyperbolic equation. Commun. Pure Appl. Math. 51, 475-504 (1998)

9. Himonas, A, Misiolek, G, Ponce, G, Zhou, Y: Persistence properties and unique continuation of solutions of the Camassa-Holm equation. Commun. Math. Phys. 271, 511-522 (2007)

10. Jiang, Z, Ni, L, Zhou, Y: Wave breaking of the Camassa-Holm equation. J. Nonlinear Sci. 22, 235-245 (2012)

11. Mckean, HP: Breakdown of a shallow water equation. Asian J. Math. 2, 767-774 (1998)

12. Zhou, Y: Wave breaking for a shallow water equation. Nonlinear Anal. 57, 137-152 (2004)

13. Zhou, Y: Blow up phenomena for the integrable Degasperis-Procesi equation. Phys. Lett. A 328, 157-162 (2004)

14. Zhou, Y, Chen, H: Wave breaking and propagation speed for the Camassa-Holm equation with $\kappa \neq 0$. Nonlinear Anal., Real World Appl. 12, 1875-1882 (2011)

15. Constantin, A, Ivanov, R: On an integrable two-component Camassa-Holm shallow water system. Phys. Lett. A 372, 7129-7132 (2008)

16. Chen, M, Liu, S, Zhang, Y: A two-component generalization of the Camassa-Holm equation and its solutions. Lett. Math. Phys. 75, 1-15 (2006)

17. Guo, Z, Zhou, Y: On solutions to a two-component generalized Camassa-Holm equation. Stud. Appl. Math. 124, 307-322 (2010)

18. Guo, Z: Blow-up and global solutions to a new integrable model with two components. J. Math. Anal. Appl. 372 , 316-327 (2010)

19. Henry, D: Infinite propagation speed for a two-component Camassa-Holm equation. Discrete Contin. Dyn. Syst., Ser B 12, 597-606 (2009)

doi:10.1186/1029-242X-2013-382

Cite this article as: Wei et al.: Infinite propagation speed for the two component $b$-family system. Journal of Inequalities and Applications 2013 2013:382.

\section{Submit your manuscript to a SpringerOpen ${ }^{\circ}$ journal and benefit from:}

- Convenient online submission

- Rigorous peer review

- Immediate publication on acceptance

- Open access: articles freely available online

- High visibility within the field

- Retaining the copyright to your article 